Review began 12/31/2021 Review ended 01/30/2022 Published 02/03/2022

\section{(๑) Copyright 2022}

Ohta et al. This is an open access article distributed under the terms of the Creative Commons Attribution License CC-BY 4.0. which permits unrestricted use, distribution, and reproduction in any medium, provided the original author and source are credited.

\title{
Acute Cholecystitis in an Elderly Patient With Antineutrophil Cytoplasmic Antibody-Associated Vasculitis: A Case Report
}

\author{
Ryuichi Ohta ${ }^{1}$, Hirotaka Ikeda ${ }^{1}$, Saya Kubota ${ }^{2}$, Chiaki Sano ${ }^{3}$ \\ 1. Community Care, Unnan City Hospital, Unnan, JPN 2. Hematology, Japanese Red Cross Society Himeji Hospital, \\ Hyogo, JPN 3. Community Medicine Management, Shimane University Faculty of Medicine, Izumo, JPN
}

Corresponding author: Ryuichi Ohta, ryuichiohta0120@gmail.com

\begin{abstract}
A diagnosis of antineutrophil cytoplasmic antibody (ANCA)-associated vasculitis is difficult to establish in elderly patients. Herein, we report a case of acute cholecystitis mimicking sepsis in an elderly patient with ANCA-associated vasculitis. A 99-year-old woman was transferred to a rural community hospital on account of anorexia and hypotension; there, she was initially diagnosed with sepsis and treated accordingly. However, she developed new-onset right upper quadrant tenderness on indirect fist percussion of the liver, and Murphy's sign was positive. While imaging did not reveal any findings suggestive of cholecystitis, the high index of suspicion for cholecystitis prompted an exploratory laparoscopy. Intraoperatively, the gallbladder wall was found to be inflamed, necessitating laparoscopic cholecystectomy. Histopathologic examination of the resected gallbladder showed neutrophilic infiltration and fibrinoid necrosis of the arterial walls. Perinuclear ANCA titers were elevated. These findings were consistent with a diagnosis of ANCA-associated vasculitis, and treatment with prednisolone markedly improved her condition. This case shows the difficulty encountered in differentiating between sepsis and ANCA-related vasculitis based on clinical features and relatively non-invasive diagnostic strategies alone. This study highlights the utility of invasive diagnostic procedures (e.g., biopsy) in elderly patients in whom a diagnosis of ANCA-associated vasculitis is difficult to establish.
\end{abstract}

Categories: Family/General Practice, Allergy/Immunology, Gastroenterology

Keywords: rural hospitals, cholecystectomy, exploratory laparoscopy, elderly, acute cholecystitis, anca-related vasculitis

\section{Introduction}

Antineutrophil cytoplasmic antibody (ANCA)-associated vasculitis is an autoimmune disease that commonly affects the skin, kidneys, lungs, and nerves [1] and presents as a rash, nephritis, pulmonary infiltration, and peripheral neuropathy [2]. Histopathologic analysis of biopsy specimens is crucial in establishing a diagnosis of ANCA-related vasculitis [3], and diagnosis can be challenging in cases where a biopsy cannot be performed. Moreover, various diseases presenting with multiorgan damage (e.g., sepsis) can mimic ANCA-associated vasculitis [4]; therefore, these differential diagnoses should be ruled out before making a diagnosis of ANCA-associated vasculitis.

The diagnosis of ANCA-related vasculitis is even more difficult in older patients owing to the various comorbidities they may have that can complicate the history and physical examination findings [5] and alter the clinical course of sepsis from acute to subacute, similar to ANCA-associated vasculitis [6]. Furthermore, owing to their immunocompromised state, elderly individuals are at a higher risk of developing sepsis [7]. While the biopsy is essential to confirm a diagnosis of ANCA-associated vasculitis, its utility in older patients is limited due to its invasiveness.

To the best of our knowledge, this is the first report of an elderly patient with cholecystitis who was pathologically diagnosed with ANCA-related vasculitis. Herein, we report a case of acute cholecystitis mimicking sepsis in an elderly patient with ANCA-associated vasculitis.

\section{Case Presentation}

A 99-year-old woman presented with anorexia and new-onset hypotension following a one-week history of fever and cough (which was left untreated), prompting admission to a rural community hospital. Her past medical history revealed hypertension, controlled with nifedipine, and osteoporosis. Prior to admission, the patient was independent in performing activities of daily living.

Evaluation of her vital signs revealed a slightly elevated temperature $\left(37.7^{\circ} \mathrm{C}\right)$ and reduced blood pressure $(96 / 62 \mathrm{mmHg}$ ) and oxygen saturation (SpO2; 92\% at room air). Heart (82 bpm) and respiratory (20 bpm) rates were normal. Head and neck examination showed no cervical lymphadenopathies. Chest auscultation revealed bilateral generalized crackles. Her abdomen was soft and non-tender. Examination of the knees and 


\section{Cureus}

hands showed no evidence of arthritis.

Laboratory results on admission are presented in Table 1 and were notable for anemia, leukocytosis, and elevated C-reactive protein levels.

\begin{tabular}{|c|c|c|c|}
\hline Laboratory Test & Day 1 & Day 7 & Reference \\
\hline White blood cells & $12.9 \times 10^{3}$ & $12.5 \times 10^{3}$ & $3.5-9.1 \times 10^{3} / \mu \mathrm{L}$ \\
\hline Neutrophils & 91.9 & 93.2 & $44.0-72.0 \%$ \\
\hline Lymphocytes & 3.9 & 2.8 & $18.0-59.0 \%$ \\
\hline Monocytes & 2.8 & 3.1 & $0.0-12.0 \%$ \\
\hline Eosinophils & 1.2 & 0.6 & $0.0-10.0 \%$ \\
\hline Basophils & 0.2 & 0.3 & $0.0-3.0 \%$ \\
\hline Red blood cells & $2.73 \times 10^{6}$ & $2.65 \times 10^{6}$ & $3.76-5.50 \times 10^{6} / \mu \mathrm{L}$ \\
\hline Hemoglobin & 7.9 & 7.5 & $11.3-15.2 \mathrm{~g} / \mathrm{dL}$ \\
\hline Hematocrit & 23.8 & 23.2 & $33.4-44.9 \%$ \\
\hline Mean corpuscular volume & 87.2 & 87.5 & 79.0-100.0 fL \\
\hline Platelets & $57.9 \times 10^{4}$ & $69.0 \times 10^{4}$ & $13.0-36.9 \times 10^{4} / \mu \mathrm{L}$ \\
\hline Total protein & 5.2 & & $6.5-8.3 \mathrm{~g} / \mathrm{dL}$ \\
\hline Albumin & 1.6 & 1.3 & $3.8-5.3 \mathrm{~g} / \mathrm{dL}$ \\
\hline Total bilirubin & 0.3 & 0.2 & $0.2-1.2 \mathrm{mg} / \mathrm{dL}$ \\
\hline Aspartate aminotransferase & 19 & 29 & 8-38 IU/L \\
\hline Alanine aminotransferase & 11 & 14 & 4-43 IU/L \\
\hline Alkaline phosphatase & 244 & 194 & $106-322 \mathrm{U} / \mathrm{L}$ \\
\hline Y-Glutamyl transpeptidase & 19 & 23 & $<48 \mathrm{IU} / \mathrm{L}$ \\
\hline Blood urea nitrogen & 17.8 & 13.7 & $8-20 \mathrm{mg} / \mathrm{dL}$ \\
\hline Creatinine & 0.85 & 0.60 & $0.40-1.10 \mathrm{mg} / \mathrm{dL}$ \\
\hline Serum Na & 139 & 138 & 135-150 mEq/L \\
\hline Serum K & 4.5 & 4.2 & $3.5-5.3 \mathrm{mEq} / \mathrm{L}$ \\
\hline Serum Cl & 106 & 104 & 98-110 mEq/L \\
\hline CRP & 30.1 & 14.76 & $<0.30 \mathrm{mg} / \mathrm{dL}$ \\
\hline Procalcitonin & 0.25 & & $0-0.05 \mathrm{ng} / \mathrm{mL}$ \\
\hline $\lg G$ & 1158 & & 870-1700 mg/dL \\
\hline $\lg \mathrm{M}$ & 40 & & $35-220$ mg/dL \\
\hline $\lg \mathrm{A}$ & 201 & & $110-410 \mathrm{mg} / \mathrm{dL}$ \\
\hline KL-6 & 313 & & 105.3-401.2 U/mL \\
\hline SP-D & 46.9 & & $<100 \mathrm{ng} / \mathrm{mL}$ \\
\hline SP-A & 31.6 & & $<31.6 \mathrm{ng} / \mathrm{mL}$ \\
\hline Leukocyte & $(-)$ & $(-)$ & \\
\hline Nitrite & $(-)$ & $(-)$ & \\
\hline Protein & $(-)$ & $(-)$ & \\
\hline Glucose & $(-)$ & $(-)$ & \\
\hline
\end{tabular}




\section{Cureus}

\begin{tabular}{lll|} 
Urobilinogen & $(-)$ & $(-)$ \\
Bilirubin & $(-)$ & $(-)$ \\
Ketone & $(-)$ & $(-)$ \\
Blood & $(-)$ & $(-)$ \\
pH & 7.0 & 6.0 \\
Specific gravity & 1.017 & 1.011 \\
\hline
\end{tabular}

\section{TABLE 1: Laboratory data on admission and Day 7 of hospitalization}

PT, prothrombin time; INR, international normalized ratio; APTT, activated partial thromboplastin time; UIBC, unsaturated iron-binding capacity; eGFR, estimated glomerular filtration rate; CK, creatine kinase; CRP, C-reactive protein

Chest radiography (Figure 1) and computed tomography (CT) (Figure 2) demonstrated bilateral pulmonary infiltrates.

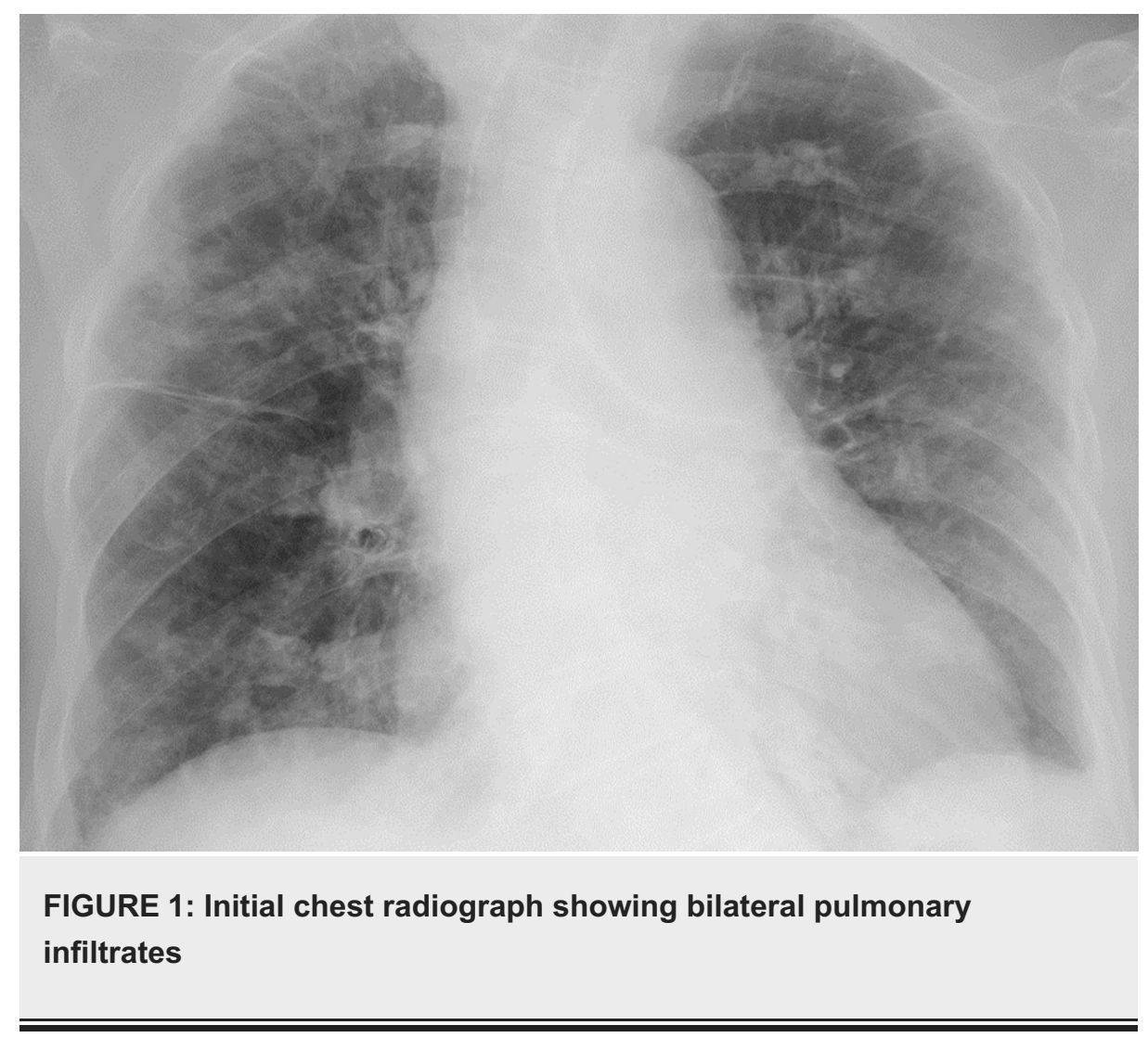




\section{Cureus}

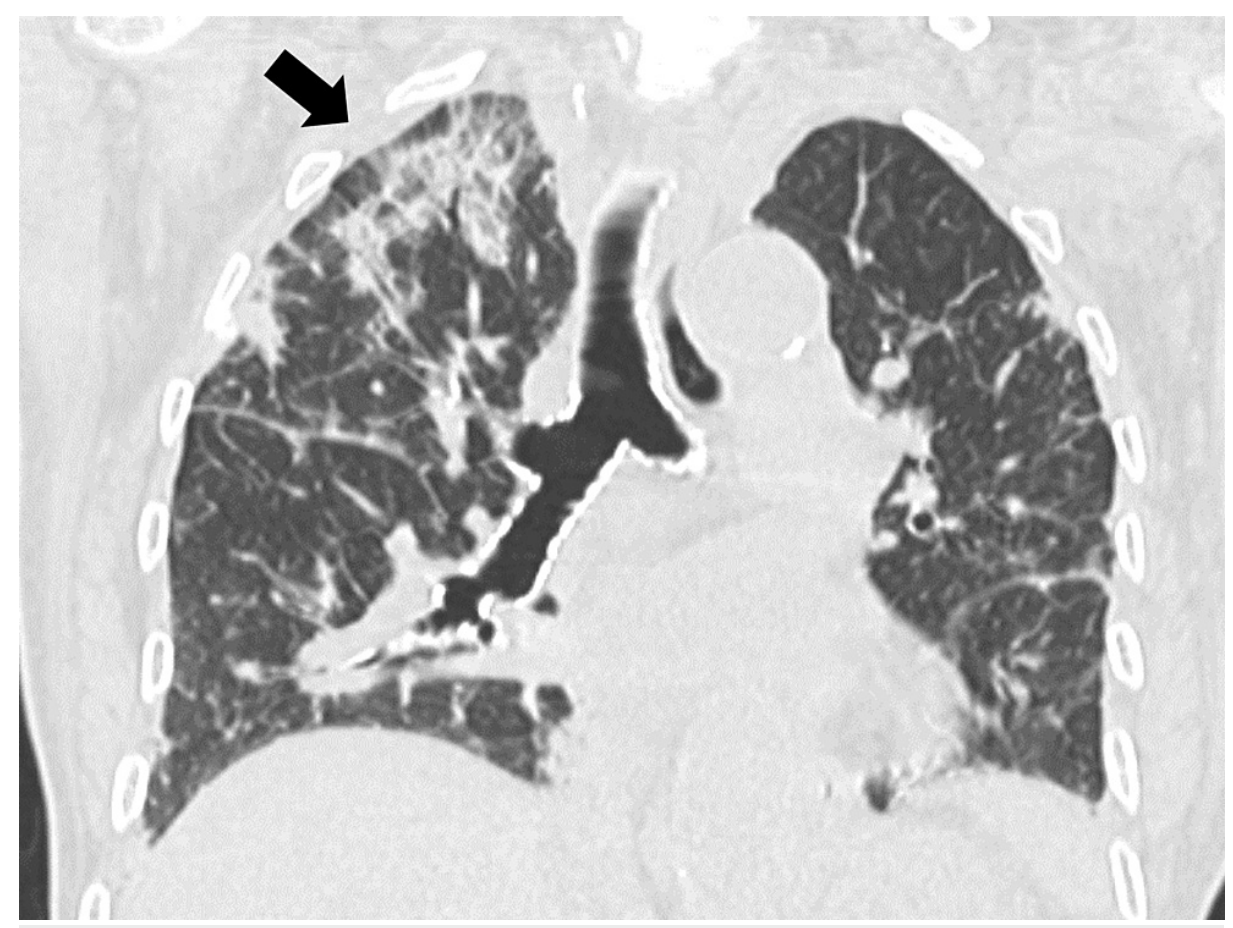

FIGURE 2: Initial chest computed tomography showing bilateral pulmonary infiltrates predominantly on the right lung

Sputum Gram staining showed neutrophilic and polymicrobial patterns. Regarding anemia, we performed an upper gastroduodenoscopy and a blood stool test, neither of which showed bleeding. We suspected interstitial pneumonia, but KL-6 and other factors indicative of interstitial pneumonia were negative, ruling out the disease. These findings were consistent with a diagnosis of bacterial pneumonia and sepsis, prompting treatment with ceftriaxone ( $2 \mathrm{~g} /$ day) and intravenous fluids. After initiation of treatment, her blood pressure normalized even though vasopressors were not used.

On the seventh day of admission, the patient developed right upper quadrant tenderness on indirect fist liver percussion and Murphy's sign was positive. Contrast-enhanced abdominal CT did not reveal any evidence of cholecystitis or cholangitis (Figure 3). 


\section{Cureus}

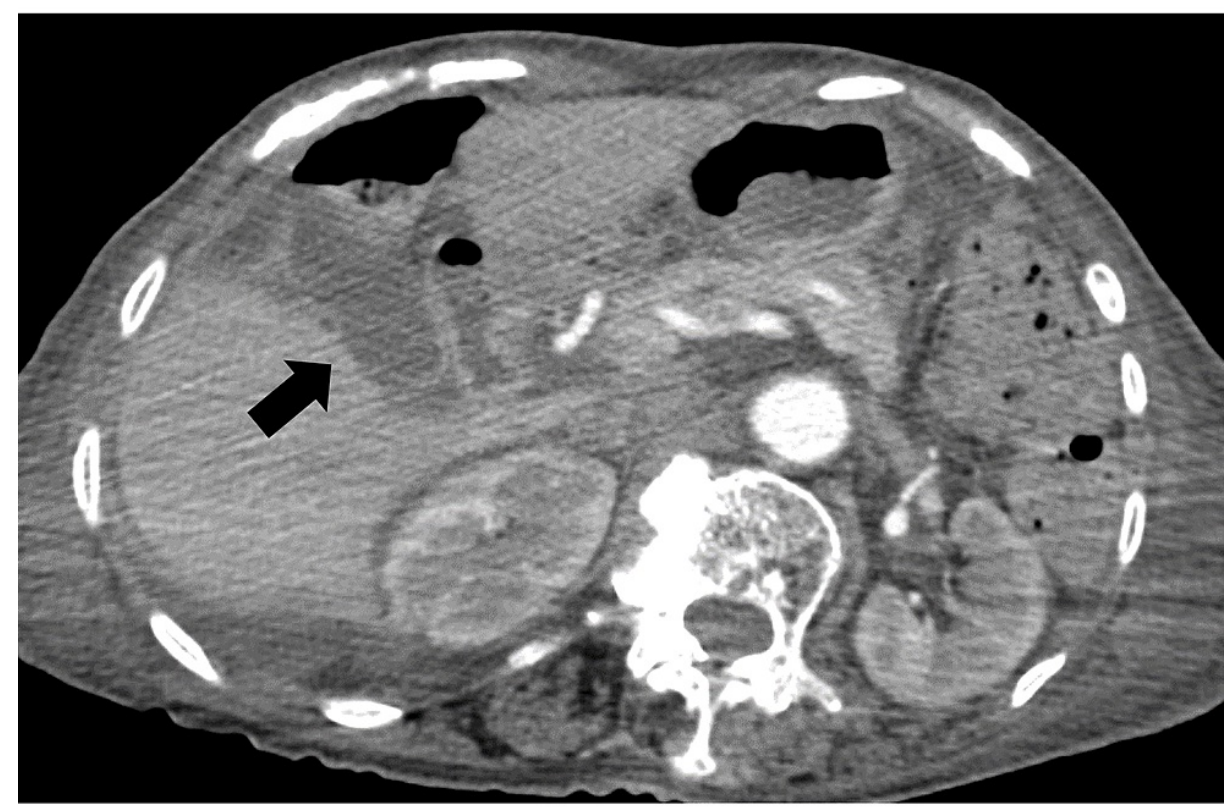

FIGURE 3: Contrast-enhanced abdominal computed tomography revealing gallbladder edema without strong wall enhancement

Liver function tests were unremarkable. Because of the absence of findings suggestive of other inflammatory lesions, we suspected cholecystitis based on the physical examination features.

The patient underwent exploratory laparoscopy, which revealed an inflamed gallbladder wall. Cholecystectomy was then performed, and no organism was detected on gram staining of bile. Histopathologic analysis of the resected gallbladder showed neutrophilic infiltration and fibrinoid necrosis of the arterial walls (Figure 4). 


\section{Cureus}
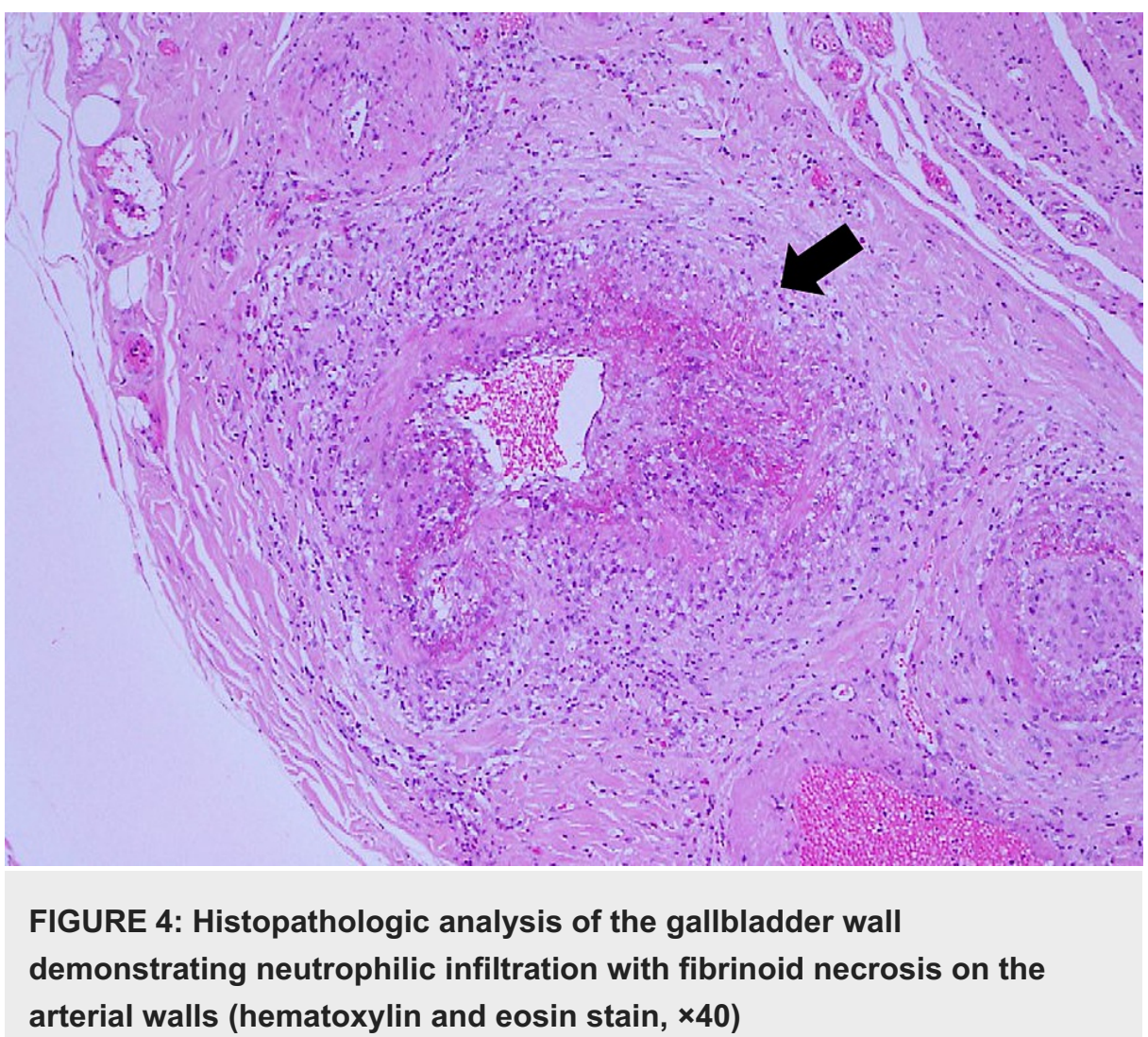

Postoperatively, the abdominal pain resolved but perinuclear antineutrophil cytoplasmic antibodies (pANCA) were elevated at $263 \mathrm{U} / \mathrm{ml}$ (normal range $<3.5 \mathrm{U} / \mathrm{ml}$ ). These findings were consistent with a diagnosis of ANCA-associated vasculitis with cholecystitis.

The patient was treated with prednisolone $(1 \mathrm{mg} / \mathrm{kg})$ and the fever resolved; we gradually tapered the drug. She was placed on a maintenance dose of prednisolone ( $3 \mathrm{mg} /$ day) for two years, and her blood pressure normalized without nifedipine. Anemia, which could have resulted from vasculitis, resolved as well (hemoglobin, $11.7 \mathrm{~g} / \mathrm{dL}$ ).

\section{Discussion}

This case shows the difficulty encountered in differentiating the history and physical examination findings of sepsis from those of ANCA-associated vasculitis. Even among elderly patients, invasive diagnostic procedures play important roles in the diagnosis and subsequent treatment of ANCA-associated vasculitis.

The differentiation between sepsis and ANCA-associated vasculitis is even more challenging in patients with complicated clinical conditions, requiring close follow-up. Both medical conditions can induce dehydration, leading to shock [8]. Elderly individuals are at higher risk of dehydration even in mild conditions, owing to the physiologic changes associated with aging [9]. Moreover, sepsis and ANCA-associated vasculitis can both cause multiorgan failure due to systemic or arterial inflammation [10]. In most cases, the degree of disease progression may help differentiate the two conditions. However, many diseases, including sepsis, have atypical clinical courses in elderly patients [11], making the diagnosis of ANCA-associated vasculitis difficult. Thus, ANCA-associated vasculitis should be considered in patients being managed for sepsis. Close follow-up of their symptoms is critical to accurately detect vasculitis.

Furthermore, although rare, the coexistence of sepsis and ANCA-associated vasculitis should be considered. In our case, the patient got slightly better with the administration of antibiotics for sepsis and pneumonia, but the fever was persistent due to vasculitis. She might have had a case of coexistence of sepsis and vasculitis. Older patients have weak immune systems, so early treatment of sepsis is vital. For the management of fever in older patients, sepsis should be treated promptly if suspected, the persistence of fever despite treatment should be investigated, and other inflammatory diseases, such as vasculitis, should be ruled out.

The clinical features of cholecystitis secondary to ANCA-associated vasculitis may differ from those of calculous cholecystitis. Calculous cholecystitis refers to the obstruction of the cholecystic duct by gallstones, resulting in an increased intracystic pressure that may cause wall ischemia and infection, affecting all layers 
of the wall of the gallbladder [12]. In contrast, cholecystitis secondary to ANCA-associated vasculitis is directly caused by gallbladder wall inflammation [13]. Because gallbladder wall inflammation in ANCAassociated vasculitis is most often focal, the typical imaging findings of cholecystitis

(e.g., gallbladder dilatation and diffuse wall thickening) are rarely seen [14]. The clinical course of calculous cholecystitis can be acute pain and consecutive fever and sepsis, which may be different from cholecystitis secondary to vasculitis, which manifests as mild abdominal pain and persistent fever of gradual onset [1213]. As such, this can delay the diagnosis of cholecystitis in patients with ANCA-associated vasculitis.

The use of a multidisciplinary approach in the diagnosis and management of acute cholecystitis in elderly patients with ANCA-associated vasculitis is very important. In this case, the surgeon was initially reluctant to perform invasive interventions on a 99-year-old patient. However, the development of clinical features consistent with cholecystitis (i.e., right upper quadrant tenderness on indirect fist liver percussion and a positive Murphy's sign) [15] strengthened the decision to push through with invasive interventions, which later revealed ANCA-associated vasculitis. This highlights the importance of going against age-related stereotypes that limit the use of invasive procedures in elderly patients [16].

\section{Conclusions}

We encountered a case of acute cholecystitis mimicking sepsis in an elderly patient with ANCA-associated vasculitis. This study illustrates the difficulty encountered in differentiating ANCA-associated vasculitis from sepsis based on clinical features and relatively non-invasive diagnostic strategies alone and highlights the utility of invasive diagnostic procedures (e.g., biopsy) in elderly patients in whom the diagnosis of ANCA-associated vasculitis is difficult to establish.

\section{Additional Information \\ Disclosures}

Human subjects: Consent was obtained or waived by all participants in this study. Conflicts of interest: In compliance with the ICMJE uniform disclosure form, all authors declare the following: Payment/services info: All authors have declared that no financial support was received from any organization for the submitted work. Financial relationships: All authors have declared that they have no financial relationships at present or within the previous three years with any organizations that might have an interest in the submitted work. Other relationships: All authors have declared that there are no other relationships or activities that could appear to have influenced the submitted work.

\section{References}

1. Kitching AR, Anders HJ, Basu N, et al.: ANCA-associated vasculitis. Nat Rev Dis Primers. 2020, 6:71. 10.1038/s41572-020-0204-y

2. Scott DG, Watts RA: Epidemiology and clinical features of systemic vasculitis . Clin Exp Nephrol. 2013, 17:607-10. 10.1007/s10157-013-0830-8

3. Ferrario F, Vanzati A, Pagni F: Pathology of ANCA-associated vasculitis. Clin Exp Nephrol. 2013, 17:652-8. 10.1007/s10157-012-0701-8

4. Gustot T: Multiple organ failure in sepsis: prognosis and role of systemic inflammatory response . Curr Opin Crit Care. 2011, 17:153-9. 10.1097/MCC.0b013e328344b446

5. Mangin D, Heath I, Jamoulle M: Beyond diagnosis: rising to the multimorbidity challenge . BMJ. 2012, 344:e3526. 10.1136/bmj.e3526

6. Samaras N, Chevalley T, Samaras D, Gold G: Older patients in the emergency department: a review . Ann Emerg Med. 2010, 56:261-9. 10.1016/j.annemergmed.2010.04.015

7. Girard TD, Ely EW: Bacteremia and sepsis in older adults. Clin Geriatr Med. 2007, 23:633-47, viii. 10.1016/j.cger.2007.05.003

8. Holliday MA: Extracellular fluid and its proteins: dehydration, shock, and recovery . Pediatr Nephrol. 1999, 13:989-95. 10.1007/s004670050741

9. Weinberg AD, Minaker KL: Dehydration. Evaluation and management in older adults. Council on Scientific Affairs, American Medical Association. JAMA. 1995, 274:1552-6.

10. Finn PJ, Plank LD, Clark MA, Connolly AB, Hill GL: Progressive cellular dehydration and proteolysis in critically ill patients. Lancet. 1996, 347:654-6. 10.1016/s0140-6736(96)91204-0

11. Girard TD, Opal SM, Ely EW: Insights into severe sepsis in older patients: from epidemiology to evidencebased management. Clin Infect Dis. 2005, 40:719-27. 10.1086/427876

12. Indar AA, Beckingham IJ: Acute cholecystitis. BMJ. 2002, 325:639-43. 10.1136/bmj.325.7365.639

13. Juliano J, Wilson KD, Gertner E: Vasculitis of the gallbladder. Case report and spectrum of disease . J Clin Rheumatol. 2009, 15:75-7. 10.1097/RHU.0b013e318194b299

14. Pérez-Vega C, Rodríguez de Castro-Hurtado E, Barrio-Molina Mdel M, Narváez J: Acute cholecystitis at initial presentation of microscopic polyangiitis. Am J Med. 2015, 128:e5-7. 10.1016/j.amjmed.2015.04.023

15. Ueda T, Ishida E: Indirect fist percussion of the liver is a more sensitive technique for detecting hepatobiliary infections than Murphy's Sign. Curr Gerontol Geriatr Res. 2015, 2015:431638. $10.1155 / 2015 / 431638$

16. Amano S, Ohta R, Sano C: Recognition of anemia in elderly people in a rural community hospital . Int J Environ Res Public Health. 2021, 18:11179. 10.3390/ijerph182111179 